\title{
Percorsi diegetici e tematici della scrittura giovanile di Elsa Morante
}

\author{
Elena PORCIANI ${ }^{1}$ \\ Seconda Università di Napoli \\ elena.porciani@unina2.it
}

\begin{abstract}
RIASSUNTO
L'articolo prende in esame le principali costanti diegetiche e tematiche dalla produzione giovanile di Elsa Morante. In particolare, il primo paragrafo rende conto delle ragioni del ritardo della critica nello studio del corpus testuale morantiano dal 1931 al 1942. Dopo che nel secondo paragrafo sono fissate le dovute coordinate metodologiche e cronologiche dello studio, il terzo si focalizza la memoria diegetica e la metanarrazione che già caratterizzano le più precoce produzione della scrittrice mentre il quarto e ultimo analizza la ricorrenza di temi e oggetti, a partire dal variegato tema del sogno.
\end{abstract}

Parole chiave: sogno, tematica, meta narrazione, oggetti, periodizzazione

A Diegetic and Thematic Itinerary into Elsa Morante's Early Works

\begin{abstract}
The article examines the main diegetic and thematic constants in Elsa Morante's early works. The first paragraph dwells upon the reasons why for a long time critics paid little attention to the texts that Morante published between 1931 and 1942. After the second paragraph is aimed at illustrating the methodological and chronological coordinates of the study, the third analyses diegetic memory and metanarration in young Morante. Eventually the forth paragraph analyses the recurrence of objects and themes, such as the theme of dream.
\end{abstract}

Key words: Dream, Thematic, Meta-narration, Objects, Division into periods

Sommario: 1. Introduzione; 2. Le ragioni dell'oblio; 3. Coordinate metodologiche e cronologiche; 4. Memoria diegetica e metanarrazione; Riferimenti bibliografici.

1 Dipartimento di Lettere e Beni Culturali, Piazza San Francesco, Complesso San Francesco, I-81055, Santa Maria Capua Vetere (Caserta), Italia. 


\section{INTRODUZIONE}

In questo contributo mi propongo di offrire una ricognizione dei principali itinerari diegetici e tematici che caratterizzano la scrittura giovanile di Elsa Morante, considerando già degna di nota la circostanza che il primo convegno tenutosi in occasione del centenario della nascita della scrittrice abbia prestato una speciale attenzione alla fase iniziale della sua opera: difficilmente sino a una decina di anni fa, prima della pur lacunosa pubblicazione dei Racconti dimenticati (Morante 2002), si sarebbero compresi nel dibattito i più di centoventi testi fra racconti, fiabe, articoli, poesie e persino un romanzo a puntate pubblicati in rivista tra il 1933 e il 1941, cui si aggiungono i volumi del Gioco segreto (1941) e delle Bellissime avventure di Cateri dalla trecciolina (1942). Nonostante si tratti di un corpus che solo per le sue dimensioni, a prescindere dal giudizio di valore, promette di essere rilevante per un'interpretazione a tutto campo della narrativa dell'autrice, a parte sporadiche eccezioni la "preistoria" morantiana (Morante 1994: 215) è stata a lungo trascurata in sede critica. Nel prossimo paragrafo cercherò di rendere conto delle ragioni di questa situazione, mentre nei successivi, dopo aver predisposto le necessarie coordinate metodologiche e cronologiche, mi dedicherò più da vicino alle costanti e varianti del tirocinio narrativo della giovane Elsa.

\section{LE RAGIONI DELL'OBLIO}

La prima ragione che ha impedito una più precoce attenzione della critica verso gli anni giovanili è stato il veto a una ricerca sistematica implicitamente posto dalla scrittrice con la sua severa selezione per la silloge del 1963: la formazione narrativa morantiana è stata per molto tempo appiattita sull'esiguo gruppo di testi presenti nello Scialle andaluso, senza riconoscere come essi in realtà appartengano a un tirocinio giovanile scandito da diversi momenti e filoni. La prima acquisizione critica degli ultimi anni è consistita, quindi, nel superamento di un approccio impressionistico e monolitico a un corpus che è senz'altro acerbo e condizionato dalle contingenze di stampa, ma non per questo è meno dotato di uno spirito di ricerca e di una volontà di mettere a fuoco la propria identità di autrice attraverso tentativi ed esperimenti che delineano un autentico laboratorio in progress.

Sul destino a lungo negletto della preistoria di Morante non meno ha pesato il fatto che sull'animo censorio della scrittrice e sulla disparità qualitativa con la produzione della maturità si è innestato l'orientamento di colui che, non solo in virtù dell'amicizia che lo legava all'autrice ma anche per alcune indubbie intuizioni interpretative, è stato a lungo il critico morantiano per eccellenza: Cesare Garboli. In particolare, hanno inciso alcune sue affermazioni che si possono leggere nella «Premessa» al secondo volume dei Meridiani, uscito nel 1990, e cioè che Morante sarebbe stata «un Autore [che], letterariamente, non si sa da dove viene» (Garboli 1995a: 19), in quanto estranea a ogni scuola e modello, e «uno scrittore precoce, ma di originalità tardiva. Precoci e irresistibili i primi passi, il bisogno di raccontare 
favole e storie; ma tardiva la rivelazione di sé a se stessa, la conquista della personalità e dello stile» (Garboli 1995a: 21). Di fronte alla certo non precocissima maturità romanzesca morantiana si può anche concordare su quest'ultimo giudizio; è però importante rendersi conto come una simile conquista non sia stata un improvviso e quasi miracoloso evento avvenuto intorno ai trent'anni, bensì il punto di arrivo di un percorso che Morante ha portato avanti per più di decennio, cosa che Garboli non sembra troppo voler ammettere: «Non è certo un caso che la stessa Morante, lei per prima, abbia silenziosamente cancellato e come sommerso le tracce di quella cospicua produzione a metà tra il feuilleton, il servizio giornalistico, il racconto di appendice, la favoletta per ragazzi, che precede la stesura del primo dei suoi romanzi» (Garboli 1995a: 25). Tuttavia, a ben vedere, le parole del critico si rivelano preziose non tanto nel difendere il veto di un'autrice che giustamente avrebbe voluto essere letta nei suoi lavori migliori più che nelle prove spesso incerte del suo tirocinio, quanto nell'indicarci - malgré lui - proprio quei modelli e generi all'interno dei quali prende origine la scrittura di Elsa Morante. E in questa direzione nell'ultima pagina dello scritto, quasi di straforo, si legge anche che Morante è «cervantesca e dostoievschiana nel demone fantastico», tesa a presupporre «la tragedia e la parodia piccolo-borghese dei romanzi di Kafka, non meno che la diagnosi derisoria dell'umanità piccolo-borghese in Madame Bovary (e magari anche in Bouvard et Pécuchet)» (Garboli 1995a: 25).

Cinque anni dopo, licenziando i saggi raccolti nel suo Gioco segreto, Garboli ha esplicitamente ammesso che quella dei racconti giovanili era «una pista da rivalutare» (Garboli 1995b: 14), come lui stesso avrebbe fatto pochi anni dopo nel saggio introduttivo ai Racconti dimenticati, significativamente intitolato Dovuto a Elsa: «Si può definire questo libro un atto dovuto. Dovuto, in primo luogo, a Elsa Morante, di cui si raccolgono qui le primissime prove di narratrice, non come reliquie ma come antefatti essenziali di una personalità letteraria così centrale nel Novecento» (Garboli 2002: V). Il critico, però, continua a mantenersi sul crinale di una formazione di compromesso tra il ripensamento e le posizioni più assertorie, come si vede nel momento in cui si rivolge ai sempre più numerosi studiosi che si sono interrogati «sulla provenienza di uno scrittore senza antenati, se si eccettuino i romanzi d'avventura o cosiddetti popolari, oltre a Verga e a un Kafka tutto e solo per sé» (Garboli 2002: V). ${ }^{2}$ Anche in questo caso, infatti, al di là dei punti programmatici, le deroghe elencate finiscono per costruire una costellazione di antenati letterari piuttosto che alimentare la tesi dell' 'autogenerazione', comunque ribadita in conclusione del discorso: "Ma è ormai chiaro come il sole che la letteratura della Morante da altri non proviene che da se stessa» (Garboli 2002: V). Si nota però tutta la torsione del ragionamento, nel quale si taglia corto per sostenere una tesi ormai divenuta paradossale: anche senza voler aggiungere Madame Bovary e Don Chisciotte, oppure Schopenhauer, Nietzsche e Freud, i vari autori e generi nominati sono di per sé sufficienti a individuare le diverse

\footnotetext{
${ }^{2}$ Corsivo mio.
} 
componenti della scrittura giovanile dell'autrice, la quale poi, mano a mano che la sua maturazione aveva corso, le avrebbe sempre più 'morantizzate' con quello stile che, certo, non altrove che dall'esperienza personale, di scrittrice e di donna, poteva provenire - ma non è poi ciò che accade con tutti i massimi autori?

In ogni caso, il titolo della raccolta postuma del 2002, Racconti dimenticatii, vuole rimarcare l'oblio che ha coperto i testi giovanili per decenni; solo che non tutto è stato recuperato: nonostante non si diano spiegazioni in merito ai criteri della scelta, solo quarantacinque lavori sono stati ripubblicati, i quali, sommati ai tredici dello Scialle andaluso e alle fiabe e filastrocche già riunite da Giuseppe Pontremoli nel 1996 per Einaudi Ragazzi (Morante 1996) ${ }^{3}$, coprono a malapena la metà circa del corpus giovanile lasciando il resto a giacere negli scaffali di poche biblioteche. E si può intuire come la presenza di così tanti testi 'abbandonati' continui a ostacolare lo studio del tirocinio che conduce dagli esordi a Menzogna e sortilegio: perché chi si occupa della produzione giovanile deve sua sponte recuperare la grande quantità di lavori distribuiti nelle riviste del tempo, pena il rischio di decontestualizzare e banalizzare con osservazioni superficiali le radici narrative di Morante. In più, si devono aggiungere alcuni lavori recentissimamente venuti alla luce, dei quali si dovrà rendere conto con la dovuta cura. Anzitutto, tra i fascicoli disponibili presso la Biblioteca Nazionale Centrale di Roma dopo l'ultima donazione degli eredi, sono stati ritrovati quattro testi 'sommersi' - la fiaba Storia di una bambina e di due bambole e i racconti Il primo amore, Il peso, Chiesa di Santa Maria. Leggenda (Porciani 2013a) - più due versioni del Ladro dei lumi che permettono finalmente di gettare luce sulla genesi di questo racconto rimasto per quasi quaranta anni assai misterioso (Porciani 2012b). Da un rapido accenno contenuto in Benedetta maledetta di Marcello Morante sono state invece rintracciate - da Marco Bardini ed Eleonora Cardinale - le poesie Tutto, Gioia, Grido dell'allodola e Saluto della sera, apparse tra il 1931 e il 1933 sulla rivista d'ascendenza futurista L'eroica. Si tratta di una riscoperta molto interessante che non solo lascia sperare di poter recuperare ancora nuovi testi dispersi nelle riviste del tempo, ma soprattutto ricolloca l'attività poetica di Elsa Morante in una posizione tutt'altro che marginale, nonostante l'esiguità delle testimonianze a stampa della giovinezza: essa «in realtà accompagna la scrittrice fin dai suoi esordi, anzi nasce in lei ancora bambina come testimoniano due quaderni scolastici dove si incontrano i suoi primi versi» (Cardinale 2012: 93; cfr. anche Bardini 2012: 125133).

${ }^{3}$ Da notare che, malgrado il libro sia intitolato come la fiaba pubblicata in volume nel 1942, Le bellissime avventure di Caterì dalla trecciolina, la versione ristampata della fiaba è quella del 1959 (Le straordinarie avventure di Caterina). 


\section{COORDINATE METODOLOGICHE E CRONOLOGICHE}

Per un articolato discorso sulla scrittura giovanile di Elsa Morante sono necessarie alcune coordinate metodologiche e cronologiche. Per quanto riguarda le prime, mi pare opportuno prendere le mosse dalla citazione di una pregnante metafora utilizzata da Giuseppe Nava che nel 1994, in uno dei più significativi contributi sul Gioco segreto, parlava di «metastasi di un antico trauma» (Nava 1994: 53) per indicare il movimento tematico che si irraggia nell'opera morantiana sin dalla prima produzione. In effetti a percorrere i testi giovanili si incontrano non solo temi, ma anche situazioni narrative, personaggi, persino oggetti ricorrenti che riappariranno nella maturità: ad esempio la rappresentazione dell'adulterio e delle gravidanze illegittime ad esso seguite, già presente, a conferma di una comprensibile urgenza tematica, nella prima pubblicazione per adulti di cui si ha notizia: Il romanzo del piccolo Bepi, uscito su Novella il $1^{\circ}$ ottobre 1933 - e, detto per inciso, dispiace che un testo così fondativo sia rimasto fuori dai Racconti dimenticati

L'immagine della metastasi, tuttavia, se appare calzante per la capacità di descrivere l'espansione magmatica delle ricorrenze tematiche, non è esente da rischi per quanto riguarda l'origine traumatica che Nava le riconosce. Questo secondo aspetto potrebbe indurre a interpretare Morante con le armi di una rigida psicocritica, instaurando rapporti di dipendenza deterministica tra la vita e l'opera, esattamente ciò di cui in sede critica la scrittrice, spesso oggetto di inarrestabili flussi di ricordi e aneddoti, ha meno bisogno. Così, ha senz'altro colto nel segno Alessandra Ginzburg quando nel saggio «Il Diario 1938 di Elsa Morante» ha ricondotto l'esperienza dell'autrice a «una ferita originaria e profonda nella relazione con la figura materna» (Ginzburg 2011: 184) che fa sì che «l'immaginario della Morante» ribalti «queste premesse dolorose nel loro contrario, fino a generare un'esperienza autocurativa che consenta di esorcizzare l'indisponibilità dell'oggetto d'amore» (Ginzburg 2011: 186); tuttavia, a mio avviso, è doveroso concentrarsi sulla dinamica più stringentemente testuale della metastasi tematica, risolvendo la contiguità esistenziale in una questione di autore implicato dai testi come nucleo di coerenza semantica più che in ipotesi interpretative sulla persona reale. 'Tematiche ossessive', quindi, più che metafore alla Mauron.

Riguardo alle coordinate cronologiche, riprendo qui la scansione che avevo già proposto nella mia monografia L'alibi del sogno nella scrittura giovanile di Elsa Morante (Porciani 2006), pur tenendo adesso conto dell'antefatto poetico, dalla patina epigonalmente dannunziana, dell'Eroica. Allo stato dei materiali conosciuti, la produzione giovanile di Elsa Morante può essere infatti ripartita in tre momenti; quello iniziale comprende gli anni tra il 1933 e il 1936, contraddistinti dalla pubblicazione da un lato di fiabe e filastrocche, soprattutto sul Corriere dei piccoli, dall'altro dei primi testi per adulti, perlopiù su I diritti della scuola. Tra questi ultimi spicca Qualcuno bussa alla porta, un romanzo in ventinove puntate, uscite tra il 25 settembre 1935 e il 30 agosto 1936, che la compresenza di elementi fiabeschi, fantastici, romanzeschi, pastorali rende, più che un pastiche, un pasticcio 
romance. Il lavoro narra le vicende di Lucia, abbandonata appena nata dalla madre zingara e adottata da un cupo aristocratico che la tiene per anni segregata in un giardino e vuole poi darla in sposa a un ricco americano, senza riuscirvi perché Lucia fugge con uno spiantato musicista, a sua volta nipote di una dark lady invecchiata e paralitica che vive nelle vicinanze. Si può facilmente intuire come il testo sia assai sconnesso, con passaggi della trama bruschi e immotivati, ma non per questo mancano gli spunti di interesse, anzi Elsa Morante dà vita a una genealogia al femminile del romanzesco in cui, al di là delle incongruenze, $i$ tre personaggi femminili - Lucia, la madre Mirtilla e la dark lady Elena - sono quasi gli unici a 'estorcere' nella sua parabola narrativa il diritto all'azione e all'avventura.

Segue il biennio 1937-1938, che segna l'inizio di una scrittura maggiormente consapevole e controllata: quattro racconti usciti sul Meridiano di Roma, che affiancano quelli pubblicati sulla rivista per maestre, l'articolo Mille città in una uscito su Prospettive e le Lettere ad Antonio, incentrate sulla trascrizione dei propri sogni. Adesso, però, i nuovi materiali relativi al Ladro dei lumi, il cui dattiloscritto conservato risale al maggio 1936, permettono di retrodatare l'inizio dell'ispirazione di questa seconda fase già all'anno precedente (Porciani 2012b), anche se le pubblicazioni prendono il via solo nel 1937 .

Elsa Morante sta entrando nella Roma letteraria che conta e il suo modo di scrivere ne risente: da una parte, si avverte la maggiore familiarità con Freud, anche se probabilmente tramite la lettura di testi divulgativi italiani più che attraverso il contatto con l'originale (Bardini 1990; Rosa 1993); dall'altra, si percepisce la curiosità verso il fantastico e il surreale, che testimonia di una vicinanza, ancora compiutamente da esplorare, con quella Italia magica che sarebbe stata dopo pochi anni antologizzata da Contini. Specialmente i racconti apparsi sul Meridiano di Roma segnano il passaggio a una narrativa dominata dal perturbante, ora declinato nel fantastico - L'uomo dagli occhiali (25/4/1937) -, ora nel fiabesco perverso - La nonna (1-8/8/1937) -, ora nel surreale-onirico - Via dell'Angelo (14/8/1938) -, ora nel melodrammatico-teatrale in quello che è forse il pezzo più pregiato del laboratorio giovanile: Il giuoco segreto (13/6/1937), destinato a diventare Il gioco segreto nella raccolta del 1941 a cui, non a caso, dà il titolo. Gli altri racconti, invece, si dividono principalmente fra testi agiografico-didattici, caratterizzati da una tematica religiosa che si unisce con l'onirico e il fiabesco - quasi a prendere il posto delle fiabe, il cui filone si sta esaurendo -, e testi che avviano la costruzione di quello che si può definire il Familienromance morantiano: la predisposizione a narrazioni familiari in cui l'ambientazione realistica si sposa con un trattamento da basso romance, laddove l'atmosfera della fiabe degli anni precedenti risultava contaminata da dettagli realistici.

Il triennio 1939-1941 è segnato da un consistente aumento del numero dei lavori pubblicati, soprattutto in ragione della regolare collaborazione al settimanale di attualità e cultura Oggi, dove appaiono, oltre ai racconti e pochi pezzi a firma propria, anche vari articoli di costume sotto lo pseudonimo di Antonio Carrera. Nel complesso, in quest'ultima fase, si riconosce la progressiva scoperta di Don Chisciotte, per dirla nei termini della stessa Morante che più volte, nel corso della 
sua vita, avrebbe ricondotto la propria narrativa a tre personaggi archetipici: Achille, Don Chisciotte e Amleto (Porciani 2013b). In particolare, nell'intervista su Le Monde a Michel David del 1968 avrebbe affermato di aver privilegiato, fra i tre, Don Chisciotte (David 1968: 8), ossia la figura del folle sognatore: di colui che di fronte alla disillusione della realtà si rifugia nel mondo privato e cristallizzato dei propri sogni, all'interno del quale, privo sia di senso della realtà che di senso del piacere, consumerà sotto la veste 'futile' del melodramma la tragedia della propria esistenza.

Negli anni precedenti non erano mancate le figure al contempo patetiche e tragiche, perlopiù femminili, di esistenze spezzate: madri adultere che si autocondannano a ogni umiliazione per espiare il peccato (Giorno di compere, I diritti della scuola, 12/5/1935) o vecchie zitelle che vivono nel ricordo del saluto giovanile di un cugino o di un ufficiale al teatro (La bella vita della vecchia Susanna, I diritti della scuola, 20/6/1935, e La vecchia, ivi, 5/12/1937). Adesso, però, questa femminilità disgraziata e umiliata viene rappresentata in testi che accentuano la separazione tra interiorità ed esteriorità nei termini di una feroce, persino superba repressione delle passioni e del desiderio, come nel caso delle sorelle nubili che si odiano pur vivendo insieme (Le due sorelle, Oggi, 16/9/1938) oppure della casalinga che vive una fastosa esistenza parallela, tutta mentale, rispetto alla miseria della sua vita reale (La moglie brutta, I diritti della scuola, 30/3/1940). Una simile accentuazione del sogno patologico a occhi aperti reca con sé due corollari: in primo luogo, tocca anche personaggi maschili privi di virilità, 'senza carattere', come recita il titolo di uno di questi racconti (Oggi, 28/6/1941); dopodiché, si unisce al recupero del repertorio basso romanzesco - filtri d'amore, maledizioni, adulteri - e alla declinazione gotica e delittuosa della passionalità folle e repressa dei personaggi, come accade nel Confessore (Prospettive, 15/1015/12/1940) e Il matrimonio del barone (Oggi, 17-24/5/1941). Colpisce infine in questo periodo la presenza di testi dalla forte connotazione umoristica, a cominciare da quella specie di autofiction a puntate che è Il giardino d'infanzia, recuperato, anche se non del tutto, nei Racconti dimenticati e recentemente ripubblicato con il titolo Aneddoti infantili (Morante 2013), nella quale la giovane autrice rivela «la gioia di prendersi sottogamba e di ridere di se stessa» (Garboli 2002: XII) rievocando episodi della propria infanzia e adolescenza.

A chiusura del periodo giovanile, nel 1941, si situa la raccolta Il gioco segreto, che costituisce la prima pubblicazione in volume, se non si conta la partecipazione come ghost writer a Scricciolo e C.i di Guelfo Civinini, prima apparso a puntate sul Corriere dei Piccoli nel 1935 e poi in volume nel 1937. Nel 1942, poi, sarebbero uscite Le bellissime avventure di Cateri dalla trecciolina, divenute nel 1959 Le straordinarie avventure di Caterina, con l'eliminazione dal titolo di quelle trecce che spesso ricorrono nella produzione giovanile, sin dalla Storia di Giovannola del '35 (Il Corriere dei piccoli, 10/2/1935) in cui la capricciosa protagonista deve sottoporsi a un espiatorio taglio di capelli per liberare la domestica tedesca Ja, imprigionata per non averle permesso di fare il bagno - ma poi si scopre che era 
solo un sogno; per trovare un vero e proprio taglio di trecce, bisognerà attendere Anna Massia in Menzogna e sortilegio.

\section{MEMORIA DIEGETICA E METANARRAZIONE}

Già da questa prima ricognizione si saranno riconosciuti alcuni temi e scenari tipici di Morante, come gli interni piccolo-borghesi, contrapposti a sporadiche escursioni nel mondo popolare, non meno misero, e nel mondo nobiliare, non meno meschino, e poi la cristallizzazione psicologica, la superbia senza senso di realtà, la mitopoiesi dell'infanzia, una prima commistione di femminile e maschile. Prima, però, di aggiungere ulteriori tasselli tematici, vale la pena di rilevare nella scansione cronologica degli anni giovanili alcuni significative metamorfosi di genere ${ }^{4}$.

Sin da ragazzina Morante si è avvicinata alla scrittura scrivendo fiabe, che, non a caso, costituiscono il genere prevalente del primo periodo giovanile, dopodiché, come si è sinteticamente visto, esse sono gradualmente sostituite dalla produzione per adulti, al punto che la pubblicazione di Caterì nel ' 42 verrà descritta, in un appunto della fine degli anni Cinquanta, come 'postuma', retaggio di una sensibilità compositiva ormai superata (Garboli-Morante 1988: XX). Questo non significa che le fiabe escano completamente dall'orizzonte poietico della scrittrice, anzi nella progressiva trasformazione da genere (fiaba) a modo (fiabesco e favoloso) sta una delle chiavi di accesso non solo alla preistoria di Morante, ma proprio alla sua intera opera. Agisce, cioè, quella che si potrebbe definire 'memoria diegetica', cosicché i generi in precedenza praticati e poi tralasciati diventano componenti dell'ipergenere morantiano in via di formazione: componenti modali, si potrebbe dire, relative a particolari modi narrativi, come il fiabesco, il romanzesco, e poi il fantastico, persino il pastorale, che si combinano tra di loro in quella dialettica di romance e novel, di tono da feuilleton e ambientazioni realistiche, che costituirà una delle costanti della narrativa morantiana più matura.

Da questo punto di vista è molto significativa una sequenza di Qualcuno bussa alla porta, dedicata al giardino del palazzo di Michele Wogan, il padre adottivo di Lucia. Il giardino costituisce, con evidenza, uno spazio fortemente simbolico, che era già apparso nella lunga Storia dei bimbi e delle stelle (Il corriere dei piccoli, 5/3-30/4/1933) nella forma di uno stellare Eden prenatale ${ }^{5}$; nel cambiamento di genere esso sembrerebbe diventato un luogo terrestre che obbedisce alle coordinate

${ }^{4}$ Doveroso riconoscere che il termine costituisce una delle intuizioni più fertili e resistenti di Garboli: «L'incessante metamorfosi che si nota di libro in libro nell'opera della Morante tende a descrivere progressivamente, nel suo arco, un sistema dotato di forte, ma anche tragica coerenza, attraversato e sostenuto da simmetrie interne» (Garboli 1990: 24).

${ }^{5}$ «Questa piccola rosa [Mariolina] cresceva in un giardino sconfinato, dove sbocciano sempre fiori di tutte le qualità. [...] Il giardino si trova su una stella. Tutti possono vedere questa stella, ma il difficile è riconoscerla, perché da lontano sembra uguale a tutte le altre» (Morante 1996: 17-18). 
della verosimiglianza, sennonché, in virtù della sua remotezza spaziotemporale, ecco che acquisisce un'aura comunque fiabesca:

Poi la carrozza entrò in un giardino, in cui gli alberi crescevano con una passione selvaggia. I lunghi pini del nord, i palmizi ondeggianti del sud e gli alberi dai frutti rotondi e caldi ombreggiavano le erbe e univano i loro forti aromi ai profumi dolciastri di fiore. Qui crescevano fiori e grappoli, di un forte lilla, e fiori dai calici molli e sfatti, pieni di polvere. E piccoli gruppi di fiori celesti e rossi, che rendono beato chi li odora. Grandi statue dal corpo mutilato fissavano l'orizzonte coi loro occhi di marmo; e ridicole scimmie, uccelli con le piume verdi, celesti e d'oro, pavoni simili ad una favola, gattini striati, si aggiravano per il giardino. Più sopra, era la montagna, in cui non vivevano che i caprioli e gli uccelli. E in basso era il mare, e le barche venute dal largo scorgevano il palazzo come un bel sogno, lontanissimo (Morante 1935/1936: 48).

Nel momento in cui la carrozza entra nel giardino, il fiabesco si contamina con un senso di distanza incantata che assume caratteri esotici: lo scenario infantile si colora di una fissità favolosa in cui il ciclo vitale si avvolge su se stesso. Non a caso, nella prospettiva delle barche che, «venute dal largo», navigano verso la costa, la lussureggiante vegetazione e la colorata fauna del giardino appaiono come «un bel sogno», a sottolineare la distanza fascinosa del luogo. A sua volta, la parvenza onirica, 'lontanissima', della terraferma entra in corrispondenza con i sogni di Lucia bambina, «meravigliosi, simili ad un mare profondo» (Morante 1935/1936: 48).

In questo precoce recupero come modalità narrativa di un genere praticato in precedenza si ha modo di vedere un primo esempio di quello che, in una prospettiva di lunghissimo periodo, costituisce il riuso del fiabesco e del favoloso nella scrittura di Morante, secondo un processo di trasformazione che arriva sino ad Aracoeli. In particolare, in una delle sequenze più estreme, ossia nell'incontro allucinatorio di Emanuele con la madre nel deserto petroso di El Amandral, si legge in riferimento al cronotopo primitivo di Totetaco, luogo e tempo favoloso: «Altro era il colore di quel sogno antico, dove lo spazio d'aria celeste e il respiro beato di Aracoeli facevano tutt'uno» (Morante 1982: 184). In questa pagina, cioè, Morante porta a compimento un itinerario tematico avviato cinquanta anni prima nelle fiabe: la nascita - e poi la crescita - come caduta, perché la vita è una continua discesa rispetto al periodo idilliaco dell'infanzia, a sua volta già esito di una precedente preistorica - cesura rispetto alla beata spensierata vita di quei non ancora nati che, nella Storia dei bimbi e delle stelle, sono senza cuore:

Tutti i bambini, quando vengono sulla terra, sanno solo il loro grido appassionato «Ov'è? $O v^{\prime} e ̀$ ? $O v ' e ̀ ? »$ e gli altri credono che cerchino il babbo, e un poeta ha creduto che cercassero il cielo, ma noi sappiamo che essi vogliono la loro stella, Ultimafata, tutto ciò che hanno perduto, e che vorrebbero ritrovare: «Ov'è? Ov'è? Ov'è? (Morante 1996: 101102).

Un altro aspetto delle metamorfosi narrative morantiane riguarda il distanziamento umoristico rispetto ai motivi e temi basso romanzeschi, come si nota 
mettendo a confronto due racconti di un certo valore che rispettivamente aprono e chiudono la terza fase della preistoria morantiana: Festa da ballo, uscito nel gennaio 1939 su I diritti della scuola, e Le ambiziose, uscito nel dicembre 1941 su Oggi, troppo tardi per essere raccolto nel Gioco segreto - una vera occasione perduta perché si tratta di un testo summa che avrebbe meritato maggiore fortuna, dato che si assiste al rovesciamento umoristico non solo del Familienromance, ma anche delle tematiche religiose sporadicamente emerse nei racconti.

In Festa da ballo - purtroppo non ripubblicato nei Racconti dimenticati - si narra la vicenda di una famiglia tutta al femminile in cui la madre, ambiziosa, compie ogni sacrificio possibile per garantire alle due figlie un futuro radioso e socialmente più elevato: le iscrive al ginnasio, fa in modo che frequentino compagne altolocate, si trasferisce con loro in un appartamento più grande nel quale una stanza sarà riservata esclusivamente ai balli che, nell'ottica della madre, dovrebbero premiare l'ascesa sociale in corso delle ragazze. Invece, nessuna festa avrà mai luogo perché le ragazze si vergogneranno comunque delle loro origini: tutto si ricoprirà di polvere e disillusione, finché le due partiranno - una sposa a un principe spiantato, l'altra attrice senza talento - e torneranno solo dopo la morte della madre:

Allora si ritrovarono insieme, loro due sole, nella loro casa antica. Tutto era in ordine, e una strana sensazione le prese: al vedere gli oggetti già noti, credettero di scorgerli come in certe fotografie sbiadite di cinquant'anni fa, senza luci e senza corpo. E insieme una tormentosa tenerezza, una domanda soffocata veniva da quegli oggetti, finché con occhi aperti e coscienti esse videro che ognuno di essi aveva significato un desiderio e un sogno, e in ognuno di essi parlava la madre. [...] Era ancora l'amore della madre che li faceva vivere; e questo amore non saziato era misto di un tale dolore, che le due figlie, di cui una spiava nell'altra i segni delle sconfitte, la bellezza già sciupata, le prime rughe, si guardarono come per accusarsi a vicenda (Morante 1939: 104).

Anche nelle Ambiziose abbiamo una famiglia tutta al femminile, ma il tono è completamente diverso, impostato su un registro che ha preso le distanze dal patetismo ottocentesco di Festa da ballo. Protagoniste sono Angela e Concetta, madre e figlia: la prima, megalomane e superba, desidera per la seconda un grandioso matrimonio che le permetta una altrettanto grandiosa ascesa sociale; la seconda prende talmente alla lettera l'ambizione della madre che si innamora niente di meno che del Signore e, ignorando ogni pretendente terreno, desidera esclusivamente congiungersi con lo Sposo Celeste e diventare la Regina del Paradiso. Per questo si fa suora e passa la sua breve vita a fantasticare sulla «magione celeste», la più splendente e meravigliosa fra tutte quelle possibili per le giovani spose; anche il delirio durante l'agonia della polmonite continua su questo tono: 
Tutte intorno sospendemmo il fiato. Ella piegava i cigli, con aria amorosa, e appena si udiva la sua voce che sospirava: «Vedo un prato di gigli, vedo i santi e gli angeli. Questo bel palazzo, è mio, Signore! Che bel palazzo, quante corone, per me...». Parve interrompersi, quasi cercasse parole di ringraziamento; la sua bocca era rimasta socchiusa, ma taceva (Morante 1941: 13). ${ }^{6}$

Non solo ogni topos mistico è stato evidentemente rovesciato, ma, per quanto riguarda più da vicino la formazione di Elsa Morante, si nota come sia l'incontro delle tragedie familiari con il racconto umoristico che, nel dicembre 1941, dà vita a quel tono melodrammatico che sarà poi decisivo nelle 'frivole tragedie' di Menzogna e sortilegio. Detto altrimenti, in questo testo conclusivo della prima lunga fase della scrittura morantiana pienamente «s'intravede la materia verista decomporsi, disfarsi sotto l'azione di un acido» (Garboli 2002: X), come ha rilevato Garboli senza però collegare tale osservazione a un'altra precedentemente messa in campo e che potrebbe illustrare che cosa sia da intendersi con tale 'acidità': che «il mondo verista della Morante contiene un demone, il suo rovescio, il sospetto che il vero sia una finzione» (Garboli 2002: VIII). Ciò significa che gli antenati ottocenteschi - Flaubert e Verga in primis, ma anche Schopenhauer - si sono finalmente armonizzati in un esito novecentesco in cui Don Chisciotte è ombreggiato da Kafka e Freud e in cui sboccia la scrittura della romanziera Elsa Morante, che d'ora in avanti assorbirà con sempre più sicura e vorace opera di morantizzazione le proprie fonti e i propri palinsesti.

Un altro filo rosso che attraversa il laboratorio giovanile è dato dal passaggio dall'eterodiegesi all'omodiegesi, che inizia a essere praticata con regolarità solo nella terza fase del '39-'41, con un progressivo avvicinamento alle scelte della maturità, assai refrattaria alla terza persona (Porciani 2012a). Se in precedenza si è considerato il primo caso (parziale) di omodiegesi il racconto La vecchia del dicembre 1937, nel quale in una lunga sequenza in prima persona la protagonista prende la parola per raccontare la storia del ventaglio che indossava quando, decenni prima, un ufficiale la salutò al teatro, adesso, dopo la scoperta dei materiali del Ladro dei lumi, si può affermare con certezza che è questo racconto a costituire la più precoce testimonianza a nostra disposizione di un io narrante. Ad esso si può affiancare, sebbene non se ne conosca con precisione la data di composizione, uno dei racconti ritrovati dell'archivio giovanile conservato presso la Biblioteca Nazionale Centrale di Roma: Chiesa di Santa Maria. Leggenda, in cui il racconto, al confine tra psicologico e fantastico, è svolto dalla stessa protagonista, per quanto ormai distante secoli dalla sua esperienza di forastica giovane suora in una città devastata dalle invasioni dei barbari e dalla dissolutezza dei cittadini.

\footnotetext{
${ }^{6}$ Cito direttamente dalla rivista perché la versione dei Racconti dimenticati riproduce la diversa versione di un dattiloscritto ritrovato tra le carte, in cui, tra l'altro, Concetta muore di tifo anziché di polmonite.
} 
Ciò non toglie che già nelle prime pubblicazioni per bambini si possa individuare un precedente dell'omodiegesi nella presenza di un io narrante che in qualche modo interviene nella storia e aggiusta le cose, spesso identificandosi con una vecchia raccontatrice. Un esempio ne è la Vecchia principessa di Paoletta diventò principessa (Corriere dei piccoli, 12/2/1933), una figura rassicurante, presente anche nella Ninna nanna della vecchietta del '34, ma destinata a essere rovesciata nell'atmosfera morbosa della Nonna. In questa direzione è in special modo interessante il caso della già menzionata Storia dei bimbi e delle stelle, in quanto il topos della vecchia narratrice prelude alla concezione della scrittura come atto riparativo, pienamente esplicitata nella Prefazione di Menzogna e sortilegio, quando apprendiamo che la narrazione di Elisa prende il via dalla triplice eredità della sua famiglia: enigma, paura e menzogna. Protagonista dei nove capitoli della fiaba è infatti una bambina non ancora nata, Mariolina, che, in attesa che la cicogna venga a condurla sulla Terra, vive sulla stella di Ultimafata insieme agli altri non nati, a differenza dei quali, però, essa ha un cuore e prova sentimenti, come l'amore struggente nei confronti del volubile e insonne Daddo, il cui nome ha tutta l'apparenza di un diminutivo di Edoardo, l'altrettanto volubile e nervoso cugino di Menzogna e sortilegio. Così, se l'amore per il dispotico Daddo da parte della servizievole e umile Mariolina, "una personcina ben educata" definita anche una «sognatrice» mette già in atto le modalità tipiche dell'amore morantiano, basate sul rapporto servo/padrone (cfr. infra), d'altro canto è interessante notare che, nel momento di massima infelicità, dopo che Daddo l'ha abbandonata per restare sulla Luna come governatore delle fate, Mariolina ricorre a una corda d'oro che la ha regalato un usignolo poeta, detto «il vecchio celibe». Non c'è un vero e proprio atto di scrittura, ma per proprietà transitiva si intuisce la funzione riparatoria dell'immaginazione e della poesia: dopo che Mariolina ha strofinato la corda d'oro fornitale dal poeta, le appare Daddo, diventato d'improvviso, per la durata di una corda, generoso e comprensivo.

\section{TEMI E OGGETTI}

Già in passato il tema del sogno mi è sembrato la linea portante della produzione giovanile morantiana, nel senso sia di esperienza onirica, sia di tutto quell'insieme di rêverie, visioni, aspirazioni, ideali in cui si articola la vasta area semantica del sogno ad occhi aperti. Si tratta di un nesso fondamentale, in quanto onirico e oniroide mettono in scena le due facce di un unico fenomeno: la possibilità di appagamento in via allucinatoria dei propri desideri da parte di personaggi che sono attesi, sul piano della realtà, da un destino di frustrazione e isolamento. Sennonché, nella maggior parte dei casi, anche l'appagamento nel mondo privato dei sogni si rivela fallimentare, come accade nella Moglie brutta. 
Il tema trova la sua rappresentazione più programmatica in quel prezioso zibaldone onirico che sono le Lettere ad Antonio del 1938, in cui convivono vari tipi di sogno: ad esempio, i 'sogni processi', che mettono in scena la pesantezza psicologica di Elsa, e i sogni più aerei e lievi che, una volta desta, la giovane autrice non può fare a meno di ammirare per il loro grado di artisticità: «Un dieci con lode, autore dei sogni» si legge a commento del sogno del 25 febbraio 1938 (Morante 1989: 42). La dialettica tra i sogni processi e i sogni artistici costituisce un'autentica lezione di metodo, non meno freudiana nel valutare gli aspetti formali di quelli relativi alla simbolica onirica; è la forma del lavoro onirico, infatti, che permette di alleggerire la pesantezza esistenziale.

Il tema del sogno è connesso da una parte alla diffusa follia dei personaggi, sospesi nel loro sogno privato che li aliena dalla realtà, a volte anche in una versione più leggera e vaporosa come nel caso della protagonista di Appuntamento (Oggi, 30/12/1939) che ogni sera a mezzanotte, tra sospiri e risatine, si incontra col suo Sogno; dall'altra, al tema del teatro, non ancora così macroscopico come in seguito, ma in grado di alimentare pericolose suggestioni in animi femminili che subiranno poi la disillusione della mancanza di talento, come accade a una delle figlie del già citato Festa da ballo. Il tema del sogno si lega poi, come lasciano intuire varie annotazioni sui sogni processi, al tema della pesantezza, presente in Morante sin dalla giovinezza: con tematizzazioni esplicite, come nel racconto adesso ritrovato $\mathrm{Il}$ peso, in cui, nei vari snodi della pur breve e concitata trama, i personaggi riflettono sulla pesantezza del corpo, ma anche nel registro della narrazione, come si nota nei testi del Meridiano di Roma e nel filone più goticoromanzesco delle pubblicazioni su Oggi.

Altro nucleo tematico ricorrente, come avranno suggerito i due racconti Festa da ballo e Le ambiziose, è quello dell'assenza della figura paterna. Lo si nota anche nella Storia dei bimbi e delle stelle se, come si è visto, nei vagiti dei neonati si crede di riconoscere la ricerca del padre. La maggior parte delle famiglie rappresentate nei racconti morantiani non ha il genitore maschile e la responsabilità dell'educazione e del mantenimento dei figli ricade tutto sulle spalle materne; e sarà questa anche una costante della Morante matura, i cui padri di famiglia generalmente rinunciano a ogni autorevolezza e presenza. Si intreccia con queste connotazioni di genere quella che si può definire l'antropologia morantiana, il fatto che secondo la scrittrice, come si legge in un articolo firmato Antonio Carrera del 1941, il mondo si divide nei Servitori e i padroni. L'autrice ci tiene comunque a precisare che non si tratta di una visione gerarchica, anzi ciascuna delle due parti ha i suoi meriti:

Qui, sospetto una cosa; l'uso delle parole "padrone" e "servo" ha finito col dare ad esse un sapore tale, che i lettori avranno la sensazione, al sentir parlare di qualità native, che io voglia alludere a qualità di ordine nobile, dignitoso, altamente onorevole, quando mi riferisco ai padroni; e di ordine inferiore, poco onorevole, quando mi riferisco ai servi. I 
lettori sono in errore: da un punto di vista non superficiale, veramente umano, oserei dire che un servitore perfetto è fatto di una materia altrettanto nobile e preziosa che un padrone perfetto. Se non, anzi, più rara giacché a formare il padrone hanno contribuito per lo più la educazione, il benessere ecc., mentre il servo non godendo di simili privilegi ha tutto il merito delle sue virtù (Carrera-Morante 1941: 11).

Una simile divisione del mondo governa, a ben vedere, anche dominanti e dominati nei rapporti di amore, che sempre in Morante assumono il valore di un assoluto esercizio di potere: dallo struggimento di Mariolina per Daddo all'obbedienza di Antonia nei confronti del bellissimo e misterioso galeotto con il quale vive la sua iniziazione all'amore in Via dell'angelo o al dispotismo del fidanzato nei confronti della romantica Virginia nei Fidanzati (I diritti della scuola, 26/2/1939), che si ravvedrà solo al momento della malattia di lei. Come si nota, di solito l'essere maschile è colui che esercita il potere, la donna colei che lo subisce, a meno che non si tratti di quel rapporto spesso morboso che lega le madri ai figli maschi, eterni adolescenti nelle loro grinfie, come nella Nonna.

Oltre ai possibili percorsi tematici, che ho cercato di sinteticamente mappare, ricorrono nei testi giovanili oggetti e motivi, se non proprio situazioni narrative, che saranno ripresi nella produzione della maturità, come le trecce: dopo la fiaba già citata di Giovannona, esse ritornano nella filastrocca Rosettina alla finestra (Cartoccino dei piccoli, 25/3/1934) o nello stesso Giuoco segreto, dove, raccogliendo i capelli lisci e sempre un po' sporchi, emananti «un lieve odore di topo», ricadono «sulle spalle» magroline e sgraziate di Antonietta (Morante 1937: 7). Altro caso è quello della finestra, oggetto di soglia per eccellenza che, introdotto nella filastrocca di Rosetta - che sta sempre alla finestra ad aspettare il figlio del re -, ricorre in altri testi, come Le due sorelle, a indicare la postura passiva della donna che attende il Principe Azzurro. Altri esempi di questo tipo possono essere poi la ricorrenza degli amori tra cugini - già nella Bella vita della vecchia Susanna, mentre «Cousinage, cousinage» scrive Antonio Carrera (Carrera-Morante 1941b: 13) -, la Vergine dispettosa che nel Guardiano della grotta (1937), trent'anni prima della Canzone popolare del Mondo salvato dai ragazzini, sussurra al mendicante appena giunto in paradiso che «è stato solo uno scherzo» (Morante 1937: 210), gli stivalini lucidi della Nonna che anticipano quelli di Cesira in Menzogna e sortilegio, la madre che arriva in città dalla campagna con il canestro in testa nella fiaba $I l$ soldato del re (Corriere dei piccoli, 27/6/1937) richiamando la visita di Alessandra a Palermo al figlio Francesco in Menzogna e sortilegio. Si fanno notare anche le prime ricorrenze di termini come «strano», «amaro», «barbaro» e colpisce, nell'Arancio (I diritti della scuola, 15/7/1938), il fatto che il protagonista aspetti la crescita dell'albero come un «meraviglioso fratello», così come farà Arturo nei riguardi del «giorno pieno, la perfezione della vita»: «aspetto sempre che il mio giorno arrivi, simile a un fratello meraviglioso con cui ci si racconta, abbracciati, la lunga noia...» (Morante 1995: 187). 


\section{RIFERIMENTI BIBLIOGRAFICI}

BARDINI, Marco (1990): «Dei "fantastici doppi" ovvero la mimesi narrativa dello spostamento psichico», in AA.VV, Per Elisa, Pisa, Nistri-Lischi, 1990: pp. 173299.

BARDINI, Marco (2012): «Elsa Morante e L'Eroica», Italianistica. Rivista di letteratura italiana, 41: pp. 121-136.

CARDINALE, Eleonora (2012): «O genio rinchiuso in una / cupola rossa ornata di papaveri»: prime osservazioni sul quaderno di Narciso, in Giuliana Zagra (a cura di), Santi, Sultani e Gran Capitani in camera mia. Inediti e ritrovati dall'Archivio di Elsa Morante (Biblioteca Nazionale Centrale. Roma, 26 ottobre 2012 - 31 gennaio 2013), Roma, Biblioteca Nazionale Centrale, 2012: pp. 93102.

CARrera, Antonio (alias Elsa MorANTE) (1941a): «I servitori e i padroni», Oggi, 18/1/1941: p. 11.

CARrera, Antonio (alias Elsa Morante) (1941b): Parenti serpenti, Oggi, 1/2/1941: p. 13.

DAVID, Michel (1968): «Entretien. Elsa Morante», Le Monde, 13/4: p. 8.

GARBOLI, Cesare (1995a): «Premessa» (1990), in Elsa Morante, Il gioco segreto, Milano, Adelphi: pp. 19-25.

GARBOLI, Cesare (1995b):«Al lettore», Elsa Morante, Il gioco segreto, Milano, Adelphi: pp.11-17.

GARBoli, Cesare / Morante, Elsa (1988): «Cronologia», in Elsa Morante, Opere, a cura di Carlo Cecchi e Cesare Garboli, Milano, I Meridiani, Mondadori, vol. I: pp. XVII-ICI.

GARBOLI, Cesare 2002 «Dovuto a Elsa», in Elsa Morante , Racconti dimenticati, a cura di Irene Babboni e Carlo Cecchi, Torino, Einaudi: pp. V-XV.

GINZBURG, Alessandra (2011): Il miracolo dell'analogia. Saggi su letteratura e psicoanalisi, Pisa, Pacini.

MORANTE, Elsa (1935/1936): «Qualcuno bussa alla porta», I diritti della scuola, 25/9/1935-30/8/1936.

MORANTE, Elsa (1937a): «Guardiano della grotta», I diritti della scuola, 25/4: p. 210.

MORANTE, Elsa (1937b): «Il giuoco segreto», Il meridiano di Roma, 13/6: pp. 7-8.

MORANTE, Elsa (1939): «Festa da ballo», I diritti della scuola, 15/1: pp. 103-104.

MORANTE, Elsa (1941): «Le ambiziose», Oggi, 6/12: pp. 12-13.

Morante, Elsa (1989a): Lettere ad Antonio (Diario 1938), Torino, Einaudi.

MORANTE, Elsa (1989b): Aracoeli , Torino, Einaudi. [Edizione originale: 1982, Torino, Einaudi]

MORANTE, Elsa (1994) «Nota», in Elsa Morante, Lo scialle andaluso Torino, Einaudi, p. 215. [Edizione originale: 1963, Torino, Einaudi] 
Morante, Elsa (1995): L'isola di Arturo, Einaudi, Torino. [Edizione originale: 1957, Torino, Einaudi]

MORANTE, Elsa (1996): Le bellissime avventure di Caterì dalla trecciolina e altre storie, a cura di Giuseppe Pontremoli, Trieste, Einaudi Ragazzi.

MORANTE, Elsa (2002): Racconti dimenticati, a cura di Irene Babboni e Carlo Cecchi, Torino, Einaudi.

MORANTE, Elsa (2013): Aneddoti infantili, Torino, Einaudi.

NAVA, Giuseppe (1994): «Il gioco segreto di Elsa Morante: i modi del racconto», in Concetta D’Angeli / Giacomo Magrini (a cura di), Vent'anni dopo La Storia, Studi novecenteschi, 21: pp. 53-78.

PORCIANI, Elena (2006): L'alibi del sogno nella scrittura giovanile di Elsa Morante, Soveria Mannelli, Iride.

PORCIANI, Elena (2012a): "Gettare il corpo nella diegesi. La Storia di Elsa Morante», in L'autore nel testo. Sette episodi di finti diari, implicature e autofinzioni, Roma, Perrone Editore: pp. 69-93.

PORCIANI, Elena (2012b): «La preistoria del Ladro dei lumi. Varianti e archeologia d'autrice», in Giuliana Zagra (a cura di), Santi, Sultani e Gran Capitani in camera mia, cit.: pp. 41-48.

PORCIANI, Elena (2013a): «Al crocevia della preistoria morantiana. I quattro testi ritrovati nelle carte giovanili», in Giuliana Zagra - Eleonora Cardinale (a cura di), "Nacqui nell'ora amara del meriggio». Scritti per Elsa Morante nel centenario della nascita, Quaderni della Biblioteca Nazionale Centrale di Roma, 17: pp. 97-110

PorCIANI, Elena (2013b): «La scelta di Don Chisciotte sulle tracce del Familienromance di Elsa Morante», Contemporanea, 11, pp. 85-96.

RosA, Giovanna 1993 «Ovvero il romanziere», in AA. VV., Per Elsa Morante, Milano, Linea d'ombra, pp. 55-87. 